\title{
Die Auseinandersetzungen zwischen dem Verwaltungsgericht Frankfurt/Main und der Exekutive über die Demonstrationsfreiheit
}

\author{
I. Negation der Demonstrationsfreibeit als Mißachtung von Demokratie und \\ Rechtsstaatlichkeit
}

Politik ist wesentlich Interessendurchsetzung auf Kosten anderer, formuliert im Namen des Allgemeinwohls. Daß sie darum ein »schmutziges Geschäft« sei, war freilich immer schon ein antidemokratisches Ressentiment derer, deren Interessen ohnedies privilegierte Durchsetzungschancen besitzen, so daß sie durchweg auf den normalen Geschäftsgang vertrauen können. Die »schweigende Minderheit« verharrt nicht in politischer Apathie, sie kann schweigen und doch auf die erfolgreiche Durchsetzung ihrer Interessen vertrauen, jedenfalls im Regelfall.

Bedürfnisse und Interessen, die im Mechanismus dieses lautlosen Regelwerks strukturell ausgeblendet und unterdrückt werden, bedürfen spezieller Artikulation und Inszenierung, um überhaupt wahrgenommen und gegebenenfalls sogar ins Kalkül gezogen zu werden. Dieser insbesondere für Minderheiten existentiell wichtige Anspruch auf politisches Gehör ist in Gestalt der politischen Freiheitsrechte, insbesondere der Meinungs-, Vereinigungs-, Versammlungs- und Demonstrationsfreiheit in Art. s, 8 und 9 des Grundgesetzes verfassungskräftig verbürgt. Wird von Seiten der Staatsorgane eines dieser Grundrechte systematisch negiert, so ist dies nicht etwa eine einmalige grundgesetzwidrige Fehlleistung, sondern ein gezielter Angriff auf eines der zentralen Strukturelemente der Verfassung: die Demokratie. Und wird solche Praxis unbeirrt fortgesetzt, obwohl sie von der Justiz wieder und wieder für offensichtlich rechts- und verfassungswidrig erklärt und aufgehoben wird, so liegt darin die Mißachtung des zweiten tragenden Elements der Verfassung: der Rechtsstaatlichkeit, derzufolge die vollziehende Gewalt ebenso wie die Rechtsprechung an „Gesetz und Recht « (Art. 20 Abs. 3 GG) sowie an die "Grundrechte . . . als unmittelbar geltendes Recht « (Art. I Abs. 3 GG) gebunden ist und staatliche Maßnahmen richterlicher Kontrolle unterworfen sind. Soviel vorweg über die politische und verfassungsrechtliche Brisanz der Auseinandersetzung zwischen der Stadtverwaltung Frankfurts (verantwortlich: Oberbürgermeister Wallmann, CDU), dem früheren Hessischen Innenminister Gries (FDP) und dem Verwaltungsgericht Frankfurt über Inhalt und Umfang der Demonstrationsfreiheit, die nachfolgend auszugsweise dokumentiert wird.

\section{Das Konfliktszenario}

Der Ablauf des Konfliktszenarios stellt sich typisiert folgendermaßen dar: Die Veranstalter melden beim Ordnungsamt der Stadt eine Demonstration an und teilen hierbei Anlaß bzw. Gegenstand, erwartete Teilnehmerzahl, Zeitpunkt und Ort bzw. Route der Kundgebung, erforderliche Zahl an Ordnern etc. mit. Wenige Tage vor der geplanten Demonstration verbietet der Oberbürgermeister als zuständige Versammlungsbehörde die Veranstaltung sowie jede Ersatzveranstaltung am gleichen 
Tag im Stadtgebiet yon Frankfurt am Main und ordnet gleichzeitig gemäß $₫ 80$ Abs. 2 Nr. 4 VwGO die sofortige Vollziehung dieser Verfügung an. Der Oberbürgermeister stützt seine Verbotsverfügung auf $\$ is Abs. I des Versammlungsgesetzes, wonach eine Versammlung verboten (oder yon bestimmten Auflagen abhängig gemacht) werden kann, wenn nach den zur Zeit des Erlasses der Verfügung erkennbaren Umständen bei der Durchführung der Versammlung die öffentliche Sicherheit und Ordnung unmittelbar gefährdet ist. Begründet wird das Verbot entweder »unter Hinweis auf Vorfälle anläßlich gleichartiger Veranstaltungen in den Vorjahren « ${ }^{1}$ mit zu befürchtenden Gewaltanwendungen unter Verweis auf Flugblätter und Aufrufe und zu erwartenden Rechtsbrüchen voraussichtlicher Teilnehmer, die ihre mangelnde Rechtstreue bereits bei vergleichbaren früheren Veranstaltungen unter Beweis gestellt hätten ${ }^{2}$ oder schlicht mit der »völligen Lahmlegung des Innenstadtverkehrs « ${ }^{3}$. Die Antragsteller legen hiergegen Widerspruch ein. Gleichzeitig beantragen sie beim Verwaltungsgericht im Eilverfahren, die aufschiebende Wirkung ihres Widerspruchs gemäß $\$ 80$ Abs. s VwGO wiederherzustellen.

Das Verwaltungsgericht Frankfurt gibt dem Antrag statt und ordnet die aufschiebende Wirkung des Widerspruchs an. Gemäß $₫ 80$ Abs. 6 S. 2 VwGO, der im Gefolge der hier dokumentierten Auseinandersetzungen zum 1. 1. 1983 gestrichen worden ist, ${ }^{4}$ war dieser Beschluß, soweit den Anträgen entsprochen ist, unanfechtbar. Dem Oberbürgermeister steht daher in diesen Fällen ein Rechtsbehelf nicht zu; auch zu einer späteren Entscheidung im Hauptverfahren (mit der Möglichkeit, beim Hessischen Verwaltungsgerichtshof Berufung einzulegen) kommt es regelmäßig nicht, weil mit der Durchführung der Demonstration die Hauptsache erledigt ist. Verläuft die Demonstration friedlich, so wird die Taktik der Polizei gelobt. Kommt es im Zusammenhang mit der Demonstration hingegen zu "Zwischenfällen « oder gar "Ausschreitungen ", so zieht die gerichtliche Entscheidung Vorwürfe auf sich.

1 Verbot der am 2. 4. 1982 angemeldeten Frauendemonstration aus Anlaß der "Walpurgisnacht « am 30. 4. 1982 durch Verfügung vom 26. 4. 1982, zit. nach VG Frankfurt, Beschluß vom 14. 5. 1982, Az: IV/I $2618 / 82$.

2 Verbot der am 25. I1. 1982 für den I 5. 5. 1982 angemeldeten Demonstration $F$ Frauen für das Demonstrationsrecht « durch Verfügung vom I 1. 5. 1982, vgl. Beschluß des VG Frankfurt vom 14. 5. 1982, Az: IV/I $2618 / 82$.

3 Verbot der am 25. I1. I98 I zum 28. 11. 1981 angemeldeten Demonstration mit Autos "Gegen Startbahn West - Volksentscheid jetzt « durch Verfügung vom 26. I1. 1981, vgl. VG Frankfurt, Beschluß vom 27. I1. 1981, Az: IV/3 $\mathrm{H} 6048 / 81$.

4 Gesetz yom 20. I2. I982, BGBI I S. I 834; dieses Dritte Gesetz zur Änderung der Verwaltungsgerichtsordnung war auf Initiative der Hessischen Landesregierung im Sommer 1982 vom Bundesrat beim Bundestag eingebracht worden mit dem Ziel, auch dem Antragsgegner - also im Regelfall der Behörde eine Beschwerdemöglichkeit für den Fall ihres Unterliegens einzuräumen (BR Drs. 122/82). Der Entwurfsbegründung zufolge zielt die Gesetzesnovelle explizit darauf, künftig solche Konfliktsituationen wie die zwischen der Exekutive und dem VG Frankfurt zu vermeiden: "Die Eröffnung der Beschwerde auch für den Antragsgegner entspricht der gewandelten Bedeutung des Verfahrens nach $₫ 80 \mathrm{Abs}$. s der Verwaltungsgerichtsordnung. Der Antragsteller wird vielfach lediglich daran interessiert sein, daß die aufschiebende Wirkung seines Widerspruchs oder seiner Anfechtungsklage angeordnet oder wiederhergestellt wird. Bei einem Demonstrationsverbot genügt es dem Veranstalter beispielsweise, wenn das Verbot nicht vollzogen werden kann, da eine Entscheidung in der Hauptsache vor der Demonstration oft nicht möglich ist. Ist diese abgeschlossen, kann er seinen Widerspruch zurücknehmen, da er sein prozessuales Ziel - die Durchführung der Demonstration - erreicht hat. In derartigen Fällen übernimmt das Verfahren nach $\$ 80$ Abs. $s$ der Verwaltungsgerichtsordnung praktisch die Bedeutung des Verfahrens in der Hauptsache. Es ist auch deshalb rechtspolitisch nicht zu vertreten, daß dem Oberverwaltungsgericht bei stattgegebenen Beschlüssen die Möglichkeit genommen wird, auf eine Vereinheitlichung der Rechtsprechung hinzuwirken."(BR Drs. x22/82) Die Erwartung, daß in der obergerichtlichen Rechtsprechung die sicherheits- und ordnungspolitischen Interessen der Exekutive stärker zum tragen kommen, hat sich in der Spruchpraxis des Hessischen Verwaltungsgerichtshofs in den durch die Gesetzesänderung ermöglichten Verfahren nach $₫ 80$ Abs. 6 VwGO denn auch prompt bestätigt. 
Von der Tatsache, daß die Verwaltung eine geplante Demonstration verbietet und das Verwaltungsgericht ihre Durchführung ermöglicht mit dem Risiko, nachträglich einer falschen Beurteilung der Situation gescholten zu werden, wäre nicht viel aufhebens zu machen, wenn es sich um gelegentliche Einzelfälle handelte. In Frankfurt häuften sich jedoch die Demonstrationsverbote zusehends:

"Laut Frankfurter Statistik gab es von 1977 bis Anfang Oktober letzten Jahres (1981, T. B.) 750 Versammlungen und Aufzüge unter freiem Himmel in der Mainmetropole, von denen ss bis 60 verboten wurden. Es wurden von den Demonstrationsveranstaltern 88 Verfahren angestrengt, um die Aufhebung von Verboten zu erzielen, und das Verwaltungsgericht Frankfurt gab in 33 Verfahren dem Antrag auf Aufhebung des Demonstrationsverbots entweder ganz oder teilweise statt" (so der damalige Hessische Innenminister Gries in der Antwort auf eine kleine Anfrage des SPD-Abgeordneten Kurth zur Demonstrationsverbotspraxis des Frankfurter Oberbürgermeisters, FAZ v. 6. r. 1982).

Seit 1979 bis Mitte Oktober 198 I wurden von 30 städtischen Demonstrationsverboten 24 durch das Verwaltungsgericht aufgehoben (laut Erklärung des Richterrats am VG Frankfurt v. I 2. I0. 198 I, vgl. FR v. I4. 10. 198 I, FAZ v. I s. 10. 1981 ), und allein in den ersten zehn Monaten des Jahres 198 I stieg die Zahl der Eilverfahren auf 21, wobei vierzehnmal den Anträgen der Demonstrationsveranstalter ganz oder teilweise stattgegeben wurde (Statistik des VG Frankfurt, unveröffentlicht). Die Stadtverwaltung spricht dagegen von 18 Verboten und 16 verwaltungsgerichtlichen Verfahren, in denen sie zwölfmal unterlegen sei (FAZ v. 19. 10. 1981). In der bereits erwähnten Antwort von Innenminister Gries auf die Landtagsanfrage Anfang 1982 liest sich dieser Sachverhalt nach übereinstimmenden Presseberichten allerdings so:

"Nach Darstellung von Gries hat es im letzten Jahr von Januar bis Ende Oktober in Frankfurt 140 Versammlungen unter freiem Himmel gegeben, von denen zwanzig unfriedlich verliefen. 82 Veranstaltungen waren angemeldet, die übrigen waren sogenannte Spontandemonstrationen. Von den 82 angemeldeten Demonstrationen wurden achtzehn verboten, und es wurde der Sofortvollzug der Verbote angeordnet. Zwei der Verbote wurden nicht angefochten, in vier Fällen hob das Verwaltungsgericht den Sofortvollzug auf. Drei dieser vier Veranstaltungen nahmen einen unfriedlichen Verlauf* (FAZ v. 6. 1. 1982, ebenso in FR v. 7. 1. 1982)

$\mathrm{Da}$ der Innenminister dieser offensichtlich unrichtigen Darstellung zufolge »keinen Grund gefunden (hat), die Demonstrationsverbotspraxis des Frankfurter Oberbürgermeisters zu beanstanden «, (FAZ v. 6. 1. 1982) verwundert nicht.

Faktisch läuft die seit 198 I drastisch verschärfte Verbotspraxis darauf hinaus, daß aus der Sicht der Stadtverwaltung in allen auch nur einigermaßen brisanten Fällen das Grundrecht der Demonstrationsfreiheit negiert wird. Damit wird die verfassungsrechtliche Regel, derzufolge die Inanspruchnahme und Ausübung einer grundgesetzlichen Freiheitsverbürgung prinzipiell zulässig ist und keiner Begründung oder gar der obrigkeitlichen Erlaubnis bedarf, auf den Kopf gestellt. Folgerichtig war die Auseinandersetzung mit dem Frankfurter Verwaltungsgericht, welche sich zum Dauerkonflikt zwischen zwei Verfassungsorganen auswuchs und seit September I98 I eine aufsehenerregende Zuspitzung erfuhr, vorprogrammiert.

\section{Die Eskalation des Konflikts seit dem Frübjabr I981}

Bereits im Frühjahr 1981 steigern sich - unter Federführung der FAZ - die Angriffe auf die Demonstrationsentscheidungen des Verwaltungsgerichts: 
"Proteste gegen die Aufhebung des Demonstrationsverbotes durch das Verwaltungsgericht haben der Einzelhandelsverband und die Industrie- und Handelskammer erhoben. Der Präsident der Universität, Professor Hartwig Kelm, wandte sich entschieden gegen die Entscheidung der Richter der IV. Kammer, den Campus der Hochschule zum Ort der Abschlußkundgebung zu bestimmen. Diese Auflage habe das Gericht erteilt, ohne die Universität zu hören. Kelm hat sich eine Aufsichtsbeschwerde vorbehalten.

Ein Sprecher des Einzelhandelsverbandes erklärte am Wochenende, seine Organisation habe kein Verständnis für die Aufhebung des Demonstrationsverbotes. Auch die vom Gericht festgelegte Route habe nur 'großes Erstaunen< hervorrufen können, da die Innenstadt dennoch erheblich beeinträchtigt worden sei. Es gebe nämlich auch ein Recht der Besucher und einkaufenden Bevölkerung auf ungehinderte Bewegungsfreiheit und Einkaufsmöglichkeiten. Die Lähmung des Verkehrs und die Behinderung der Passanten nehme aber ein solch unerträgliches Ausmaß an, daß sie nicht mehr durch das Recht auf Demonstrationen gedeckt werden könnten.

Es sei nicht länger zu vertreten, daß die Bevölkerung und auch die Einzelhandelsbetriebe in dieser Weise geschädigt würden. Geschäfte würden stark beeinträchtigt und müßten schließen, das Publikum werde am Einkauf gehindert.«(FAZ v. I6. 3. 198I).

Tags drauf, am 17. 3. 1981, hakt die FAZ nach mit der Meldung, der Präsident der Universität habe sich

»noch einmal nachdrücklich gegen die Entscheidung des Frankfurter Verwaltungsgerichts gewandt, den Campus der Hochschule ohne Rücksprache mit ihm zum Ort der Abschlußkundgebung der Demonstration vom Wochenende zu bestimmen «.

Wenig später weiß sie aus dem Munde eines Geschäftsinhabers in der Innenstadt zu berichten:

") Wissen Sie, wenn die deutsche Demokratie in Gefahr wäre und für ihre Rettung demonstriert werden sollte, würde ich vielleicht meinen Laden schließen und in einem Demonstrationszug auf der Straße mitmarschieren. Warum aber dreihundert Leute wegen der Verhältnisse in Chile samstags nahezu die gesamte Innenstadt lahmlegen können, weil ein Gericht trotz vorsorglichen Verbots ihres Protestzugs durch den Oberbürgermeister ihnen das möglich macht, das kann ich nicht verstehen`, Diese Ansicht vertritt nicht allein der Inhaber eines Fachgeschäfts am Roßmarkt. Er weiß sich mit vielen Kollegen einer Meinung, auch mit den Käufern, die, zumal an Samstagen, zu Tausenden in die Stadt kommen, um ihre Besorgungen in Ruhe zu erledigen « (FAZ v. 7.4. 1981).

Im gleichen Artikel verhilft die FAZ der neuesten Kreation des Einzelhandelsverbandes, dem "Recht auf ungestörten Einkauf «, welches dem Demonstrationsrecht mindestens (!) gleichzusetzen sei, gekoppelt mit abermaliger Kritik am Frankfurter Verwaltungsgericht, zu publizistischer Wirksamkeit:

"Der Einzelhandelsverband hat inzwischen auch festgestellt, daß ein Teil der Kunden, so er nicht ein bestimmtes Spezialgeschäft aufsuchen will, an Demonstrations-Samstagen nach Wiesbaden, Mainz oder Darmstadt zum Einkauf ausweicht. Der Verband spricht davon, daß es möglich sein müsse, das Recht des Bürgers auf freie Bewegung in der Stadt und damit auch das Recht auf ungestörten Einkauf besser als bisher zu sichern. Dieses Recht sei mindestens dem Demonstrationsrecht gleichzusetzen. Die Bemühungen der Stadt, hier abzuwägen, wenn Interessenkollisionen zu erwarten seien, erkennt der Handel und - nach Auffassung Bikkerles auch der Bürger zum überwiegenden Teil an. Die Kritik des Verbandes richtet sich gegen die wiederholten Entscheidungen der Verwaltungsgerichte, die Verbotsverfügungen der Stadt immer wieder aufhoben, so daß dem Handel, den Bürgern und letztlich auch der Stadt, die schließlich die Gewerbesteuer kassiere, Nachteile entstünden. (FAZ v. 7. 4. 1981)

Vollends ins Schußfeld der Kritik - jetzt auch von Seiten der Stadtregierung - gerät das Verwaltungsgericht Frankfurt, nachdem am 13.9. 1981 im Anschluß an einen "zunächst friedlich verlaufenen Fackelzug«, einen "für Frankfurter Verhältnisse noch ruhigen Demonstrationszug" (FAZ v. 15.9. 198I) eine Reihe Schaufensterscheiben eingeworfen werden und es zu vereinzelten Auseinandersetzungen mit der Polizei kommt. Auch hier war die Demonstration von der Stadt verboten worden und konnte erst auf Grund der Entscheidung des Verwaltungsgerichts vom I I. 9. 
198 I (Az: IV/3 H 3910/8 I) stattfinden. Tags darauf gibt der Rechtsdezernent Stadtrat Brück folgende Presseerklärung ab:

»DDie schweren Ausschreitungen im Frankfurter Westend während einer Demonstration am späten Sonntagabend hätten ebenso wie die Sachbeschädigungen vermieden werden können<. Mit diesen Worten kommentierte Frankfurts Rechtsdezernent Stadtrat Wolfram Brück den Verlauf einer Fackelzug-Demonstration durchs Westend und Bockenheim, die das Frankfurter Ordnungsamt am Freitag zunächst verboten hatte. Mit einer Entscheidung vom Freitagabend hatte das Verwaltungsgericht dieses Verbot dann wieder aufgehoben.

Brück bedauerte in diesem Zusammenhang, daß das Gericht den in der Verbotsverfügung aufgezählten Gründen nicht folgen mochte. Im Nachhinein hat sich gezeigt, daß Oberbürgermeister Dr. Walter Wallmann leider Recht behalten hat‘, so Brück, >denn wie erwartete kam es während der Demonstration zu Ausschreitungen und Sachbeschädigungen an mehreren Objekten, die zu einem Gesamtsachschaden von über 100000 Mark geführt haben.« « $[\ldots]$

"Auch in Zukunft wird der Oberbürgermeister sicher dabei bleiben «, so Stadtrat Brück abschließend, »daß er bei entsprechender Kenntnis der Zusammenhänge solche Demonstrationen verbieten wird, im Interesse unserer Mitbürger und zur Vermeidung hoher Sachschäden. «s

Die liberale Judikatur des Verwaltungsgerichts fest im Visier, so scheint es, mochte der Rechtsdezernent über juristische Zwirnsfäden nicht stolpern: Obwohl die Zeitungen übereinstimmend berichteten, daß die Auseinandersetzungen erst nach der offiziellen Beendigung der Kundgebung und in einiger Entfernung von ihr begannen (vgl. die Berichte in FAZ v. I 5. 9. 1981 und FR vom I s. 9. 1981), liest sich dies in der Presseerklärung anders. Danach fanden die »schweren Auseinandersetzungen ... während einer Demonstration « statt, und weil es so eindringlich klingt, muß es sogleich wiederholt werden: „wie erwartet kam es während der Demonstration zu Ausschreitungen «. Nicht unerwartet kam, daß sie wiederum die FAZ - wenn auch nicht in bezug auf diese "feine Unterscheidung Ausschreitungen während oder nach einer Demonstration « (auf die sich auch OB Wallmann später laut FAZ "nicht einlassen möchte «, FAZ v. 29. 10. 1981) - der ganzen Angelegenheit annahm und in einem Kommentar das rechte Demonstrationsverständnis verkündete.

"Die paar hundert Teilnehmer dieser seltsamen >Demonstration`, die zu nachtschlafender Zeit durch die Straßen Bockenheims gegangen waren, um - von wem eigentlich? - die Abschaffung der Nato zu fordern und den amerikanischen Außenminister Haig als , Gangster zu beschimpfen, waren fast völlig unter sich. . . Demonstration hatte ursprünglich den Sinn von sauf etwas aufmerksam machen< (demonstrare), wobei Fackelzüge allerdings nicht nur demokratische Tradition haben. Aber auf leeren Straßen umherziehen und geistlose Parolen ins Dunkle zu rufen, ist eine Absurdität, die den Namen Demonstration nicht mehr verdient" (1 5.9. 1981).

Solch permanente und geharnischte Urteilsschelte lockte schließlich auch die Richter der kritisierten IV. Kammer des Frankfurter Verwaltungsgerichts aus der standesüblichen Reserve:

"In ungewöhnlich scharfer Form haben ... der Präsident des Verwaltungsgerichts Frankfurt, Dieter Neumeyer, und sein Richterkollege Torsten von Roetteken die Demonstrationsverbotspraxis von Oberbürgermeister Walter Wallmann kritisiert ... Neumeyer und von Roetteken, der an der Gerichtsentscheidung mitgewirkt hatte, wiesen entschieden Brücks Vorwurf zurück. Ein Fackelzug sei angemeldet worden, und die Stadt habe keinerlei konkrete Hinweise dargeJegt, daß es seitens der Veranstalter, drei Komitees, die sich für Freiheit in El Salvador, Nicaragua und Guatemala einsetzen, zu Gewalttätigkeiten kommen könnte. Tatsächlich stand in der Verbotsverfügung lediglich, daß Einzelpersonen oder Gruppen sich, wie bereits bei der El-Salvador-Demonstration am 31. Januar dieses Jahres, anschließen und Gewalt ausüben könnten.

Sowohl Neumeyer als auch von Roetteken machten klar, daß sie nach Recht und Gesetz eine angemeldete Demonstration nur dann als Richter nicht zulassen dürfen, wenn zu befürchten 
ist, daß vom Veranstalter selbst Gewalttätigkeiten ausgehen könnten. Bei der Demonstration gegen den Haig-Besuch hätte die Stadt dazu weder etwas Konkretes vorgetragen, noch habe sich im nachhinein bestätigt, daß die Veranstalter für die Ausschreitungen verantwortlich zu machen seien.

Im Verwaltungsgericht in der Adalbertstraße in Bockenheim sieht man in diesem Verbot Wallmanns nur ein Glied in einer Kette von gesetzeswidrigen Demonstrationsverboten.

Eigentlich, meinten die beiden Richter, müßte Wallmann, denke er logisch und konsequent, auch eine Veranstaltung von Franz Josef Strauß in Frankfurt verbieten, wenn zuvor schon einmal Gruppen eine Kundgebung des bayerischen Ministerpräsidenten gestört hätten. sSollte Wallmann selbst dieses Schicksal auch ereilen«, merkte von Roetteken an, >müßte er eben auch seine eigene Veranstaltung verbieten.

Nicht länger, so Neumeyer und von Roetteken, sei man gewillt, das Spiel Wallmanns mit seinen Verboten, das von einem großen Teil der Bevölkerung sicherlich sehr wohlwollend kommentiert werde, stillschweigend hinzunehmen. Auch Oberbürgermeister Walter Wallmann sei als Vertreter der Exekutive verpflichtet, Grundgesetz und hessische Verfassung zu achten. Dem handele er zuwider.

,Wir würden ja unseren Verfassungsauftrag als dritte Gewalt verletzen<, sagte von Roetteken, swenn wir solche Verbote nicht aufheben würdenк.

Im Endeffekt würden durch die Wallmannsche Schneliverbotspraxis ohne Rücksicht auf Recht und Gesetz Exekutive und Legislative an Glaubwürdigkeit und Vertrauen einbüßen.« (FR, 16. 9. 1981)

Dies mochten nun der Oberbürgermeister und sein Rechtsdezernent nicht unwidersprochen hinnehmen. Am darauf folgenden Tag berichtet die Presse von einer erneuten Stellungnahme des Stadtrats Brück:

"Die Demonstrationsverbote, die der $\mathrm{OB}$ in den vergangenen Jahren verfügt habe, seien nicht verfassungswidrig.

,Selbstverständlich،, sagte Brück, rist das Demonstrationsrecht garantiert, aber es darf andere Rechte nicht einschränken. Der Oberbürgermeister habe auch die Rechte der Mitbürger zu vertreten, die sich an solchen Aufzügen nicht beteiligten. Im einzelnen nannte der Rechtsdezernent das Recht auf freie Entfaltung der Persönlichkeit, das Recht auf Schutz des Eigentums und auf körperliche Unversehrtheit.

Immer wieder zeige sich, daß durch vorher absehbare Ausschreitungen bei zunächst verbotenen Demonstrationen enorme Schäden zu Lasten einzelner Bürger entstünden. . . . Die politische Verantwortung hierfür<, so Brück, stragen aber nicht die Richter, die solche Demonstrationen genehmigen, sondern die trägt allein der Oberbürgermeister.

Er dürfe nicht zulassen, daß zum Schaden des größeren Teils der Frankfurter Bevölkerung , Chaoten die Genehmigungspraxis der Verwaltungsgerichte ausnutzen, um im Fahrwasser zunächst friedlicher verlaufender Demonstrationen anschließend ihr zerstörerisches Unwesen zu treiben<...

Wallmann müsse jeweils die allgemeine Sicherheitslage berücksichtigen . Komme er in Abstimmung mit den Sicherheitsbehörden zu dem Schluß, daß eine konkrete Gefahr bestehe, eine Demonstration werde gewalttätig verlaufen, dann müsse er eben verbieten.

Abschließend meinte Brück: , Sache der Richter ist in einem soichen Fall lediglich, zu überprüfen, ob diese Schlußfolgerungen aus der allgemeinen Sicherheitslage sachgemäß erfolgt sind. Die Verwaltungsrichter können nicht an Stelle des Frankfurter Oberbürgermeisters eine solche Entscheidung treffen. Das ist nun mal alleine Sache der Verwaltung. " (FR, 17. 9. 1981, vgl. auch den Bericht in FAZ v. 17.9. 1981)

Nunmehr schaltete sich auch die SPD-Fraktion der Frankfurter Stadtverordnetenversammlung in den Konflikt ein und kritisierte das Bestreben des Oberbürgermeisters, die Verantwortung für die Gewährleistung des Grundrechts auf Demonstrationsfreiheit von sich abzuwälzen. Dies widerspreche ebenso wie die »offensichtliche Oberflächlichkeit « der Verbotsverfügungen unserer Rechtsordnung (FR 17. 9. 1981; FAZ, 17. 9. 1981).

Das Klima der Auseinandersetzungen verschärft sich erneut, nachdem es nach dem Tod des jugendlichen Demonstranten Klaus Jürgen Rattay in Berlin bei einer Spontandemonstration in der Frankfurter Innenstadt am 22. 9. 1981 abermals zu erheblichen Beschädigungen kommt. Am 24. 9. I98 I wird auf ausdrückliche Weisung des Oberbürgermeisters eine für Samstag, den 26. 9. 198I angemeldete Demonstration 
"Gegen die Kriminalisierung politischer Widerstandsbewegungen « verboten. Oberbürgermeister Wallmann erklärt, daß er sich nach den Ausschreitungen in den vergangenen Tagen und Wochen "in tiefer Sorge um die Sicherheit der Bürger « (FR v. 25. 9. 1981) verpflichtet fühle, erneut der Eskalation von Gewalt entgegenzutreten. Gleichzeitig kündigte Rechtsdezernent Brück an, daß die Stadt den Präsidenten des Verwaltungsgerichts, Neumeyer, und seinen Richterkollegen von Roetteken wegen Besorgnis der Befangenheit für den Fall ablehnen würden, daß sie an einer Gerichtsentscheidung über das Verbot mitwirken sollten. Denn sie hätten die Verbote des Oberbürgermeisters » in einer unerträglichen Weise unsachgemäß kommentiert « und würden diesem »in einer ungeheuerlichen Weise sachfremde Motive unterstellen * (FR v. 25. 9. $198 \mathrm{I}$ ).

Tags drauf findet vor der IV. Kammer des Verwaltungsgerichts wie erwartet das Eilverfahren über das Demonstrationsverbot vom 24. 9. I98 I statt. Dabei stellt die Stadt erstmals die bereits in der Presse angekündigten Ablehnungsanträge gegen die Richter Neumeyer und von Roetteken, die sie auf die zitierten Presseveröffentlichungen vom 16.9. 1981 stützt. Die Befangenheitsanträge werden abgelehnt, die sofortige Vollziehbarkeit des Demonstrationsverbots jedoch aufrecht erhalten, wenn auch mit anderer Begründung als von der Stadt vorgetragen (Az: IV/3 H 4340/8 I). Trotz des Verbots kommt es zu schweren Auseinandersetzungen zwischen Polizei und Demonstranten. Obwohl das Verwaltungsgericht die Verbotsverfügung des Oberbürgermeisters im Ergebnis bestätigt hat, erneuert Rechtsdezernent Brück in einer Presseerklärung der Stadt die Vorwürfe gegenüber dem Verwaltungsgericht:

"Das von Oberbürgermeister Wallmann angeordnete Verbot der für Samstag geplanten Demonstration habe der Polizei die Handhabe gegeben, rechtzeitig und mit Erfolg gegen jene gewalttätigen Demonstranten aus der extremen linken Szener vorzugehen. Dies erklärte am Sonntag Rechtsdezernent Wolfram Brück, der zugleich den Polizisten für ihren Einsatz dankte. Die Ausschreitungen bestätigen nach Ansicht des Stadtrats Wallmanns Verbotsentscheidung. Ferner rügte Brück das Verwaltungsgericht, das in seiner Begründung, *keine konkreten Hinweise auf eine Gefährdung der öffentlichen Sicherheit und Ordnung، gesehen habe. Zu einer eigenen Lagebeurteilung, so Brück weiter, sei das Gericht snicht aufgerufen<. Der städtische Rechtsdezernent kündigte an, Wallmann werde auch in Zukunft seine Verbotspraxis fortsetzen." (FR v. 29. 9. 198I)

Am 29. 9. I98 I fordert der Vorsitzende der CDU-Stadtverordnetenfraktion, Moog, in einer Stellungnahme vor dem CDU-Arbeitskreis Frankfurter Polizeibeamter die Verlegung von Bereitschaftspolizei nach Frankfurt und spart nach Presseberichten ebenfalls nicht mit Kritik an den Demonstrationsentscheidungen des Verwaltungsgerichts (FR y. 30. 9. 198I). Tags drauf erläßt der Oberbürgermeister eine Verbotsverfügung gegen eine für Samstag, den 3. 10. 198 I angemeldete Demonstration des Allgemeinen Studentenausschusses (ASTA) der Universität Frankfurt unter dem Motto: „Berlin - Frankfurt: Jetzt ist Schluß! «. Wiederum wird das Verwaltungsgericht angerufen, wiederum stellt die Stadt in der am 2. I0. I98 I stattfindenden Sitzung - mit der gleichen Begründung wie zuvor - Ablehnungsantrag gegen Richter von Roetteken.

Das Verwaltungsgericht ändert diesmal gegenüber dem Eilverfahren vom 25. 9. 198 I sein Vorgehen: Beide Entscheidungen werden gleichzeitig erst nach schriftlichem Absetzen und Ausfertigen den Beteiligten bekanntgegeben und ausgehändigt. Der Befangenheitsantrag wird abermals abgelehnt, dem Antrag des ASTA stattgegeben (dem späteren sachlichen Vorbringen der Stadt wird durch einen Abänderungsbeschluß hinsichtlich der Demonstrationsroute gemäß $₫ 80$ Abs. 6 VwGO Rechnung getragen). Noch am gleichen Abend reagiert die Stadt mit folgender Presseerklärung: 
"I. Das Verwaltungsgericht hat sich mit seiner Entscheidung über die eindeutigen Hinweise auf eine Gefährdung der öffentlichen Sicherheit und Ordnung hinweggesetzt. Es nimmt mit seiner Entscheidung mögliche schwere Nachteile für die Frankfurter Bürger in Kauf. Es wird allein der Polizei auferlegt, mit der dadurch entstandenen schwierigen Situation fertig zu werden.

2. Das Verwaltungsgericht hat zudem in seinen Auflagen einen Demonstrationsweg festgelegt, der einen Zusammenbruch des Verkehrs am verkaufsoffenen Samstag zur Hauptverkehrszeit befürchten läßt.

3. Das Verwaltungsgericht hat der Stadt nach der erneuten Ablehnung des Richters von Roetteken keine Gelegenheit mehr gegeben, Anträge zu stellen oder Erklärungen zur Sache abzugeben. [...]

6. Die Tatsache, daß der Stadt jeder Sachvortrag abgeschnitten wurde und die Behandlung des Befangenheitsantrages wird die Stadt dazu veranlassen, Dienstaufsichtsbeschwerde beim Präsidenten des Verwaltungsgerichtshofs in Kassel einzulegen.«

Fast wäre man geneigt die Tatsache, daß die Demonstration dann doch ohne Zwischenfälle verlief, aus der Sicht der Stadtverwaltung als bedauerlich zu bezeichnen jedoch konnte dafür ja auf ein bekanntes Muster zurückgegriffen werden:

"Dem Einsatz der Polizei sei es zu verdanken, sagte gestern Rechtsdezernent Wolfram Brück, daß die Demonstration ohne Zwischenfälle verlaufen sei. [...]« (FAZ, 5. 10. 1981)

Offensichtlich entschlossen, den Konflikt auf die Spitze zu treiben, stellt die Stadtverwaltung am 6. 10. $198 \mathrm{I}$ in einem weiteren Verfahren vor der 4. Kammer gleich gegen alle drei an der Entscheidung beteiligten Richter (Richter Neumeyer, von Roetteken, Richterin Kohl) erneute Ablehnungsanträge wegen Befangenheit unter Bezugnahme auf die Presseerklärungen sowie die verfahrensmäßige Handhabung am 2. 10. 198I, wobei insoweit sogar der Verdacht der Rechtsbeugung geäußert wird (FAZ v. 29. IO. 198I). Wiederum werden die Befangenheitsanträge abgelehnt, diesmal nach mündlicher Verhandlung als unzulässig, weil verspätet vorgebracht. In der Sache selbst wird die Klage zurückgenommen. (Az: IV/I E 995/8I).

In dem Eilverfahren ASTA gegen Stadt Frankfurt wegen des Verbots einer vor der Justizvollzugsanstalt Preungesheim angemeldeten Demonstration am folgenden Tag wiederholt sich der Vorgang: Die abermals gestellten Befangenheitsanträge werden unter Hinweis auf die hierzu bereits ergangenen Entscheidungen als unzulässig zurückgewiesen, dem Antrag des ASTA unter Änderung des Kundgebungsortes stattgegeben $(\mathrm{Az}$ : IV/2 H $5180 / 8 \mathrm{I})$. Die Veranstaltung verläuft ohne Zwischenfälle angesichts des Kräfteverhältnisses verständlich: „Unzählige Mannschaftswagen der Polizei, ein Hubschrauber und - zehn Demonstranten « (FR v. 8. IO. I98I).

Kurz darauf macht die folgende Erklärung des Richterrats am Verwaltungsgericht Frankfurt, mit dem die Personalvertretung der 26 Verwaltungsrichter den bedrängten Kollegen der IV. Kammer am I 2. 10. 198 I zu Hilfe eilte, Schlagzeilen:

"Die IV. Kammer des Verwaltungsgerichts in Frankfurt am Main sieht sich in jüngster Zeit fortgesetzter Angriffe seitens namhafter Vertreter der Stadt Frankfurt am Main wegen ihrer Rechtsprechung zum Versammlungsrecht ausgesetzt. Da in diesem Zusammenhang unzutreffende Behauptungen in die Presse gelangt sind, sieht sich der Richterrat des Verwaltungsgerichts zu folgender Erklärung veranlaßt:

1. Die zahlreichen Demonstrationsverbote der Stadt Frankfurt am Main haben in der Öffentlichkeit den falschen Eindruck erweckt, als seien Demonstrationen genehmigungsbedürftig, und zwar letztlich durch die Verwaltungsgerichte. Demgegenüber räumt Artikel 8 des Grundgesetzes unmißverständlich allen Deutschen das Recht ein, sich ohne Erlaubnis friedlich zu versammeln. Auf Grund des Versammlungsgesetzes besteht für die Verwaltungsbehörde lediglich die Möglichkeit, bei Vorliegen bestimmter Voraussetzungen eine Versammlung zu verbieten. Stellt diese Entscheidung sich als rechtswidrig heraus, wird sie durch das Gericht aufgehoben und wieder der verfassungsmäßige Zustand hergestellt. Es ist somit zwar möglich, von einer Verbotspraxis der Verwaltung zu sprechen, nicht aber von einer Genehmigungspraxis des Verwaltungsgerichts, da sich die Zulässigkeit einer Versammlung aus dem Grundgesetz selbst ergibt. 
2. Bei der Anordnung eines Demonstrationsverbots durch die Verwaltungsbehörde handelt es sich nicht um eine gerichtsfreie Ermessensentscheidung. Ein Verbotsermessen steht der Behörde gemäß $\$$ is Abs. I Versammlungsgesetz nur zu, wenn bei Durchführung der Versammlung »nach den zur Zeit des Erlasses der Verfügung erkennbaren Umständen die öffentliche Sicherheit und Ordnung unmittelbar gefährdet ist«. Die Verwaltungsbehörde muß ihren Verboten also eine gerichtlich voll nachprüfbare Prognoseentscheidung über den erwarteten unfriedlichen Verlauf einer Versammlung zugrunde legen. Die gerichtliche Überprüfung der Verbotsverfügungen der Stadt Frankfurt am Main hat in der Vergangenheit in den meisten Fällen ergeben, daß die zur Begründung der Prognose vorgelegten Fakten unzureichend waren. Eine solche Entscheidung kann gegenüber dem Recht aus Artikel 8 Grundgesetz keine Geltung beanspruchen; daran kommt kein Gericht vorbei. Eine statistische Betrachtung der gerichtshängig gewordenen Verbotsverfügungen der Stadt Frankfurt am Main seit 1979 zeigt, daß in 30 Verfahren 24 Verbote aufgehoben werden mußten; dabei ist es wäbrend der Demonstrationen selbst in keinem Fall zu unfriedlichen Handlungen gekommen.

3. Das Versammlungsrecht sieht keine Möglichkeit vor, mit Blick auf »die allgemeine Sicherheitslage ein Verbot auszusprechen. Gewalthaft verlaufende Demonstrationen in anderen deutschen Städten geben der Verwaltung kein Recht, sozusagen vorsorglich Versammlungen im eigenen Zuständigkeitsbereich zu verbieten. Ebensowenig darf das Verhalten von Rowdies, Chaoten und Provokateuren ohne weiteres dem jeweiligen Veranstalter zugerechnet werden; man denke nur an die andernfalls etwa auch Fußballgroßveranstaltungen drohende Verbotsgefahr. Es bedarf vielmehr in jedem Einzelfall erneut konkreter Feststellungen über unmittelbar bevorstehende Gefahren. Unsorgfältige Ermittlungen seitens der Verwaltung wirken sich als Rechtsmangel ihrer Verfügung aus.

4. Es ist nicht Aufgabe von Gerichten, sich an Spekulationen über den politischen Hintersinn einer rechtsfehlerhaften Praxis von Demonstrationsverboten zu beteiligen. Nachdenklich sollte es aber alle Beteiligten stimmen, wenn der Eindruck entsteht, Dauerverbote und dagegen zu erwartende Rechtsmittel sollten den Gerichten in der Öffentlichkeit die alleinige Verantwortung für evtl. Zwischenfälle bei Demonstrationen aufbürden. Auf das Schärfste zurückgewiesen werden müssen schließlich presseöffentliche Äußerungen von Magistratsmitgliedern bzw. Stadtverordneten, die dem Verwaltungsgericht eine Mitverantwortung selbst in solchen Fällen von Gewaltausschreitungen anlasten, in denen das Gericht mit überhaupt keiner Entscheidung befaßt war (Freßgasse am 22.9. 1981) oder wo sogar ein Verbot der Stadt bestätigt wurde (Flohmarkt am 26. 9. 1981). Es ist daher zu wünschen, daß unter den Verantwortlichen der Stadt Frankfurt am Main der Ton der Mäßigung wieder die Oberhand gewinnt.«

(Zit. nach der Presseerklärung; vgl. auch die Berichte in FR v. 14. 10. 1981, FAZ v. 15. 10. 198I)

Wiederum läßt die Reaktion der Stadtregierung nicht lange auf sich warten: Am 1 5. I0. I981 erhebt das Rechtsamt die bereits in der Presseerklärung vom 2. I0. I981 angekündigte Dienstaufsichtsbeschwerde gegen die drei Richter der IV. Kammer des Verwaltungsgerichts, über deren Inhalt die Presse offensichtlich voll unterrichtet wurde und breit berichtet (FAZ, 23. 10. 1981 ; FR, 27. 10. 198 I FAZ 29. 10. 198 I). Vorgeworfen wird zum einen den Richtern Neumeyer und von Roetteken, daß sie sich vor der Presse grundsätzlich zur Verbotspraxis des Oberbürgermeisters geäußert hatten ("pauschale und undifferenzierte Wertung der städtischen Überlegungen « - »Unterstellung, daß Oberbürgermeister und Ordnungsamt sich regelmäßig über geltendes Recht hinwegsetzten«), zum andern allen drei Richtern (also auch Richterin Kohl), daß sie in dem Eilverfahren am 2. 10. 198 I entgegen der Prozeßordnung - und einer entsprechenden telefonischen Mitteilung von Richterin Kohl nicht zunächst über die Befangenheitsanträge befunden hatten, bevor sie in der Sache entschieden. Dadurch sei der Stadt die Gelegenheit zu Stellungnahmen in der Hauptsache genommen worden. Und schließlich argwöhnt der Rechtsdezernent insgesamt, daß es der vierten Kammer darum geht, "aus diesseits nicht erkennbaren Gründen Demonstrationen zu ermöglichen, die nach begründeten Erkenntnissen die Gefahr schwerer Ausschreitungen in sich bergen « (FAZ v. 23. I0. 1981). 
Doch allen Anfeindungen zum Trotz, die sich in der Stellungnahme des Oberbürgermeisters Wallmann auf eine dringliche Anfrage der SPD-Opposition im Stadtparlament unvermindert fortsetzen (vgl. den Bericht in FAZ v. 29. 10. 198I), bleibt die IV. Kammer des Frankfurter Verwaltungsgerichts ihrer demokratisch-rechtsstaatlichen Linie in der Demonstrationsrechtsprechung treu. Dabei scheut sie sich freilich nicht - soweit von der Sache her einsichtig - die Durchführung von Demonstrationen mit bestimmten und jeweils spezifischen Auflagen zu verbinden. Als Beispiel hierfür sei der im folgenden auszugsweise wiedergegebene Beschluß vom 27. I I. $198 \mathrm{I}$ (betr.: Autokorso »wegen Startbahn-West - Volksentscheid jetzt « Az: IV/ $3 \mathrm{H}$ $6048 / 8 \mathrm{I}$ ) angeführt:

" $[\ldots]$

II.

Einer formellen Entscheidung über die Ablehnungsgesuche der Antragsgegnerin bedarf es nicht. Die in diesem Verfahren angebrachten Ablehnungsgesuche stellen sich nämlich als Wiederholung bereits zurückgewiesener Ablehnungsgesuche dar. Dies stellt eine mißbräuchliche Rechtsausübung dar. Über sie darf das Gericht in der ordnungsgemäßen Besetzung, also ohne Einschaltung der Vertreter entscheiden (vgl. BVerwG Buchholz $310 \$ 54$ VwGO Nr. 10; RGZ 44, 402; BVfGE II, I/3; 343/348/).

Das Begehren des Antragstellers auf Gewährung einstweiligen Rechtsschutzes ist als Antrag auf Wiederherstellung der aufschiebenden Wirkung seines Widerspruches gemäß $\$ 80 \mathrm{Abs.} 5$ $\mathrm{V}_{w} \mathrm{GO}$ zulässig...

Der Antrag hat in der Sache Erfolg, da sich die angegriffene Verfügung der Antragsgegnerin bereits bei summarischer Prüfung als offensichtlich rechtswidrig erweist und Grundrechte des Antragstellers verletzt. Die Voraussetzungen für den Ausspruch eines Versammlungsverbotes gemäß $\ 15$ VersammlungsG liegen nämlich nicht vor.

Gemäß $\$ is Abs. 1 VersammlungsG darf die Antragsgegnerin als Versammlungsbehörde erst dann ein Versammlungsverbot erwägen, wenn eine unmittelbare Gefährdung der öffentlichen Sicherheit und Ordnung nach den Erkenntnissen, wie sie im Zeitpunkt des Erlasses der Verfügung vorliegen, droht. Davon kann jedoch im vorliegenden Falle keine Rede sein. Ausweislich der Verwaltungsvorgänge wie insbesondere der Verfügungsbegründung selbst befürchtete nämlich die Antragsgegnerin lediglich ganz allgemein, daß ein Autokorso mit 300 Autos, der sich über eine Länge von $2,4 \mathrm{~km}$ bis $3,0 \mathrm{~km}$ erstrecken würde, zu einem völligen Zusammenbruch des gesamten Straßen- und Fußgängerverkehrs führen werde. [...] Nach alledem ist lediglich die Gefahr zu besorgen, daß es zeitweise zu einem Zusammenbruch des Straßenverkehrs kommt. Dabei geht die Antragsgegnerin jedoch zu Unrecht davon aus, daß der vom Antragsteller geplanten Veranstaltung Rechte anderer Verkehrsteilnehmer aus Art. 2 Abs. I GG entgegenstünden. Denn diese Rechte bestehen ausweislich des Textes dieser Grundrechtsnorm nur vorbehaltlich der Rechte anderer. Das Recht auf freie Entfaltung der Persönlichkeit wird damit von verfassungswegen durch die Rechte anderer, zu denen auch das Recht auf Demonstrationsfreiheit gemäß Art. 8 Abs. I GG gehört, beschränkt. Die Rechte anderer, hier der Demonstrationsteilnehmer, finden ihrerseits eine Schranke im Recht der freien Entfaltung der Persönlichkeit erst dann, wenn dieses Recht in seinem Kernbereich verletzt wird und somit die Wesensgehaltsgarantie nach Art. I9 Abs. 2 GG eingreift. Beschränkungen der freien Entfaltung der Persönlichkeit, die in einer zeitlichen Behinderung des Auto-Straßenverkehrs liegen, stellen jedoch keine derartige Kernbereichsverletzung dar. Denn die Fortbewegung dieser Bürger ist durch die bestehenden öffentlichen Verkehrsmittel ebenso gewährleistet wie durch die Fortbewegungsmöglichkeit zu Fuß. Erst die Gleichzeitigkeit eines Zusammenbruchs des gesamten Straßen- und Fußgängerverkehrs stellt einen grundrechtsrelevanten Eingriff in das Recht auf freie Entfaltung der Persönlichkeit dar und hebt die Rechte dieser Bürger auf die gleiche Stufe wie das Demonstrationsrecht nach Art. 8 Abs. I GG. Erst in diesem Falle ist Raum für eine Abwägung zwischen den Interessen der Demonstranten und denjenigen, die sich auf ihr Recht auf freie Entfaltung der Persönlichkeit berufen. Erst die grundrechtsrelevante Verletzung des Rechtes auf freie Entfaltung der Persönlichkeit stellt eine Verletzung der öffentlichen Sicherheit und Ordnung im Sinne des $\$ is Abs. I VersammlungsG dar und rechtfertigt gegebenenfalls Eingriffe auf der Grundlage dieser Norm. [...]

Auf eine eventuelle Erlaubnis nach $₫ 29$ StVO kann es ebenfalls nicht ankommen, da öffentliche 
Versammlungen und Aufzüge keiner verkehrsrechtlichen Erlaubnis bedürften (vgl. Jagusch, 25. Aufl., $\$ 29$ StVO Rdn. 4). Dies ergibt sich schon daraus, daß der Bundesgesetzgeber im Versammlungsgesetz die Beschränkungen der grundrechtlich gewährleisteten Versammlungsfreiheit, wie sie nach Art. 8 Abs. 2 GG zulässig sind, abschließend geregelt hat. Da weder das StVG noch die StVO selbst - bei ihr handelt es sich lediglich um eine untergesetzliche Rechtsverordnung - entsprechend dem Gebot des Art. 19 Abs. 1 S. 2 GG das Grundrecht auf Versammlungsfreiheit als beschränktes oder beschränkbares Grundrecht benennen und zitieren, muß davon ausgegangen werden, daß die Vorschriften des StVG wie diejenigen der $\mathrm{StVO}$ so auszulegen und zu handhaben sind, daß sie das Grundrecht auf Versammlungsfreiheit nicht tangieren. Die Unterstellung des Grundrechts der Versammlungsfreiheit unter ein Verbot mit Erlaubnisvorbehalt würde die bundesgesetzliche Entscheidung für eine ausschließlich repressive Eingriffsmöglichkeit unterlaufen und damit der Zielsetzung des VersammlungsG als der einschlägigen Spezialregelung zuwiderlaufen.

Die angegriffene Verfügung erweist sich auch insoweit als offensichtlich rechtswidrig, als sie sich auf die Kroatendemonstration, ausgehend vom Baseler Platz, bezieht. Denn insoweit verstößt die Verfügung jedenfalls gegen den verfassungsrechtlichen Grundsatz der Verhältnismäßigkeit, der im Rechtsstaatsprinzip in Verbindung mit Art. 1 Abs. 3 GG ruht. Da die Auflagenverfügung bezüglich der Kroatendemonstration vom selben Tage datiert wie diejenige, die gegen den Antragsteller ergangen ist, wäre es der Antragsgegnerin ohne jeden Zweifel möglich gewesen, im Rahmen ihrer Ermessensbetätigung eventuelle Kollisionspunkte zwischen beiden Veranstaltungen durch den Ausspruch geeigneter Auflagen zu vermeiden. Der Ausspruch einer Verbotsverfügung gegen den Antragsteller war jedenfalls nicht erforderlich, zumal die Antragsgegnerin durch die Wahl der Auflagen gegenüber der Kroatendemonstration selbst erst die möglichen Kollisionspunkte mit der Anmeldung des Antragstellers vom 25. I I. 198 I geschaffen hat.

Auch die von der Antragsgegnerin im Verlaufe des einstweiligen Rechtsschutzverfahrens nachgeschobenen Gründe und Erwägungen lassen bis auf einige untergeordnete Punkte keine andere Entscheidung als diejenige der Wiederherstellung der aufschiebenden Wirkung zu. [...] Zwar ist der Antragsgegnerin zuzugeben, daß insoweit nunmehr eine unmittelbare Gefährdung der öffentlichen Sicherheit und Ordnung zu besorgen ist, somit also die Möglichkeit einer Verfügung nach $\$$ Is Abs. 1 VersammlungsG grundsätzlich besteht. Denn der Antragsteller hat es auch mit seinem Schriftsatz vom 27. I I. 1981 wie der dazu überreichten eidesstattlichen Versicherung nicht vermocht, die Vermutung der Antragsgegnerin auszuräumen, daß es geplant sei, die unangemeldete Demonstration an der Katharinenkirche im Bereich der Hauptwache mit dem Autokorso zusammenzuführen. Ob dies die Absicht des Antragstellers oder der Veranstalter des Autokorso selbst ist, kann dabei dahinstehen, da aufgrund der von der Antragsgegnerin überreichten dienstlichen Erklärung (Bl. 28 d. A.) gleichwohl zu befürchten ist, daß sich Teilnehmer des Autokorso, die auch die gleichen inhaltlichen Ziele wie der Antragsteller verfolgen, an der Hauptwache dazu entschließen können, sich der Demonstration an der Katharinenkirche anzuschließen anstatt ihre Fahrt fortzusetzen. Da damit neben dem Autoverkehr im Bereich der Hauptwache aufgrund der zu erwartenden großen Zahl der sonstigen Demonstrationsteilnehmer (ca. 15 000) auch eine Lahmlegung des Fußgängerverkehrs zu befürchten steht, muß von einer Gefahr für die öffentliche Sicherheit ausgegangen werden, wie bereits oben dargelegt. Diese kann jedoch entsprechend den Verhältnismäßigkeitsgrundsätzen schon dadurch ausgeräumt werden, daß der Autokorso auf der vom Antragsteller hilfsweise vorgeschlagenen Route geführt wird, da dadurch jede Berührung zur Hauptwache ausgeschlossen wird. Der Fußgängerverkehr kann damit nicht mehr beeinträchtigt werden, soweit es den Autokorso angeht. [...]

\section{Die Räumung des Hüttendorfs auf dem Gelände der Startbabn 18 West des Frankfurter Flughafens und die Konfrontation mit dem Hessischen Innenminister Gries}

Wenige Wochen vor dieser Entscheidung hatte die IV. Kammer des Frankfurter Verwaltungsgerichts in einem anderen Eilverfahren zu beschließen. Daraus entwikkelte sich ein ebenso langanhaltender wie erbitterter Konflikt mit dem damaligen hessischen Innenminister Gries, hinter dem die Auseinandersetzung mit der Stadtverwaltung - zumindest in der publizistischen Aufmerksamkeit - deutlich zurück- 
trat. Den Anstoß gab der nachfolgend auszugsweise wiedergegebene Beschluß vom 2. 11. 198I (Az: IV/2 - H s768/8I), mit dem die Kammer die undatierte (!) Verfügung des Polizeipräsidenten von Frankfurt am Main zur unverzüglichen Räumung des Hüttendorfs auf dem Waldgelände der vorgesehenen Startbahn i 8 West des Frankfurter Flughafens für »offensichtlich rechtswidrig" erklärte und die aufschiebende Wirkung des Widerspruchs der Antragsteller (der Kirchenvorstand der evangelischen Kirchengemeinde Mörfelden-Walldorf sowie einzelne seiner Mitglieder) anordnete:

"Gründe:

I.

Die Antragsteller wenden sich gegen eine undatierte Verfügung des Polizeipräsidenten in Frankfurt am Main, die gerichtet ist san die Personen, die sich im Hüttendorf aufhalten, das in dem Waldgebiet errichtet wurde, das für den Bau der geplanten Startbahn I 8 West vorgesehen iste.

In dieser Verfügung wird dem so bezeichneten Personenkreis aufgegeben, ,die illegal errichteten Hütten sofort und auf Dauer zu räumen und sich hinter die polizeiliche Absperrung zu begeben<. Für den Fall, daß die Adressaten dieser Aufforderung nicht unverzüglich nachkommen, wird die Durchsetzung der Verfügung durch Anwendung unmittelbaren Zwangs angedroht. [...]

Gegen diese Verfügung legte der Bevollmächtigte der Antragsteller mit Schreiben vom 2. I I. 1981 Widerspruch ein. Zur Begründung fügte er eine Durchschrift des Antrags gleichen Datums auf Anordnung der aufschiebenden Wirkung dieses Widerspruchs bei.

Dieser Antrag ist bei Gericht am 2. 11. 1981, gegen I 5.45 Uhr, eingegangen. [...]

Auf einen telefonischen Hinweis des Gerichts, es beständen Bedenken hinsichtlich der Antragsbefugnis der Antragstellerin zu I), hat der Bevollmächtigte der Antragsteller den Eilantrag zusätzlich im Namen der Mitglieder des Kirchenvorstandes der evangelischen Kirchengemeinde Mörfelden-Walldorf, der Antragsteller von 2) bis 6), persönlich gestellt.

$[\ldots]$

Auf Grund einer telefonischen Anfrage des Gerichts an den Polizeipräsidenten, worauf er seine Zuständigkeit zum Erlaß der angefochtenen Verfügung stütze, hat er durch einen Vertreter mitteilen lassen, seine Zuständigkeit folge aus $₫ 76$ Abs. 3 S. 3 HSOG, da der Regierungspräsident in Darmstadt mit Verfügung vom $2 \mathrm{r}$. 4. I98 I mit sofortiger Wirkung die Aufgaben im Zusammenhang mit der Startbahn West dem Polizeipräsidenten in Frankfurt am Main übertragen habe. Im einzelnen seien genannt die Vorbereitung aller notwendigen Einsatzmaßnahmen, die Durchführung erforderlich werdender Einsätze, auch in den unmittelbar an das Flughafengelände angrenzenden Gebieten.

Weitere Auskünfte hat der Antragsgegner, auch im Verlaufe weiterer telefonischer Anfragen des Gerichts, nicht erteilt.

II.

1.

Hinsichtlich der Antragstellerin zu 1) ist der Antrag mangels Antragsbefugnis unzulässig. Es ist nicht ersichtlich, inwieweit die evangelische Kirchengemeinde Mörfelden-Walldorf als solche durch die angegriffene Verfügung in eigenen Rechten betroffen sein könnte. [...]

Eine Verletzung eigener Rechte kommt nur für einzelne Mitglieder der Antragstellerin zu r), die sich auf dem in der Verfügung bezeichneten Gelände aufhalten und somit Adressaten der Verfügung sind, in Betracht, so daß die Antragsbefugnis der Antragsteller zu 2) bis 6) vorliegt.

$[\ldots]$

2.

Hinsichtlich der Antragsteller zu 2) bis 6) ist der gemäß $\$ 80$ Abs. s VwGO zulässige Eilantrag auch begründet. [...]

Nach summarischer Überprüfung der Sach- und Rechtslage hält die Kammer die angegriffene Verfügung für offensichtlich rechtswidrig.

So ist fraglich, ob der Polizeipräsident in Frankfurt am Main sachlich zuständig war. Denn es erscheint zweifelhaft, ob es sich um eine unaufschiebbar zu beseitigende Störung der öffentlichen Sicherheit und Ordnung im Sinne des $\$$ I Abs. 2 HSOG handelt. Das Hüttendorf, dessen Existenz den Antragsteller zum Einschreiten veranlaßt hat, besteht schon seit vielen Monaten. Ebenso ist der Planfeststellungsbeschluß zugunsten der FAG seit 1980 sofort vollziehbar. Seit dieser Zeit konnte mit den Bauarbeiten, die jetzt in Angriff genommen werden sollen, jederzeit begonnen werden. Ebenso war seit dieser Zeit damit zu rechnen, daß Gegner der geplanten 

zu behindern.

Es ist nicht ersichtlich - weder aus dem Inhalt der angegriffenen Verfügung, noch aus einem Vortrag des Antragsgegners im Eilverfahren - inwiefern die Ausgangslage für die mit den Aufgaben der Gefahrenabwehr betrauten Behörden sich zu einem bestimmten Zeitpunkt kurzfristig so entscheidend verändert hat, daß ein Einschreiten der nach \ I Abs. 3 HSOG zuständigen allgemeinen Verwaltungsbehörde - hier der Bauaufsichtsbehörde nach $\$ 83$ Abs. 1 HBO - nicht mehr möglich war.

Diese hätte nämlich neben dem Abbruch der formell und materiell illegalen Gebäude des Hüttendorfes auch ein Nutzungsverbot aussprechen und ggf. die sofortige Vollziehung anordnen können. Warum all dies unterblieben ist und nun nicht mehr nachgeholt werden konnte, ist weder dargelegt noch sonst ersichtlich.

Es ist auch zweifelhaft, ob die Verfügung des Regierungspräsidenten mit dem Inhalt, der dem Gericht von dem Antragsgegner telefonisch mitgeteilt wurde, überhaupt auf $\$ 76$ Abs. 3 S. 3 HSOG gestützt werden kann. Soweit eine generelle Übertragung von Maßnahmen der Gefahrenabwehr im Zusammenhang mit dem Bau der Startbahn 18 West beabsichtigt war, wäre als Rechtsgrundlage $\$ 75$ Abs. 2 HSOG einschlägig. $\$ 76$ Abs. 3 S. 3 HSOG ist weder nach seiner systematischen Stellung noch dem Sinnzusammenhang der Regelung des $\$ 76$ HSOG nach auf die Übertragung einer Sonderzuständigkeit im Einzelfall zugeschnitten. [...]

Im Hinblick auf die in der Begründung der Verfügung dargelegten bereits begangenen strafbaren Handlungen ließe sich die Zuständigkeit des Polizeipräsidenten nach $\$ \mathrm{r}$ Abs. 2 HSOG ebenfalls nicht begründen, da insoweit die Zuständigkeit der Strafbehörden vorliegt; hier greift das HSOG, welches präventiv-polizeiliche Maßnahmen regelt, nicht mehr ein.

Falls man von einer Zuständigkeit des Polizeipräsidenten im Rahmen der Leistung von Vollzugshilfe $(\$ 44$ Abs. 3 HSOG) zur Durchsetzung von Rechten der FAG aus dem Planfeststellungsbeschlu $ß$ ausginge, wäre die angegriffene Verfügung offensichtlich nicht geeignet, den mit ihr erstrebten Zweck zu erreichen. Nach der Begründung der Verfügung sollte mit ihr erreicht werden, daß diejenigen, die sich im Hüttendorf aufhalten - wobei von der Bestimmbarkeit dieses Personenkreises ausgegangen werden soll - nicht die beginnenden Bauarbeiten an der Okrifteler Straße behindern. Offensichtlich kann aber eine Person, die sich in einer der Hütten des sog. Hüttendorfes aufhält, nicht zugleich die Baumaßnahmen an der Okrifteler Straße behindern.

Dabei verkennt die Kammer nicht, daß das mit der Verfügung angestrebte Ziel des Antragsgegners vermutlich war, durch die Räumung der Hütten die Gefahr auszuschalten, daß das sog. Hüttendorf als Sammelpunkt für Gegner der geplanten Baumaßnahmen dienen konnte. Der Antragsgegner hat weder in der angegriffenen Verfügung noch im Rahmen des Eilverfahrens dargelegt, daß die sich in den Hütten aufhaltenden Gegner des Startbahnbaus, solange sie sich in den Hütten aufhalten, Störer im polizeirechtlichen Sinne bzgl. der Bauarbeiten an der Okrifteler Straße sind. Eine präventive Entfernung potentieller Störer, soweit sie im vorliegenden Zusammenhang allein dem Zweck dienen soll, die Sicherung der Bauarbeiten an der Okrifteler Straße - in einiger Entfernung vom Hüttendorf - zu erleichtern, würde aber gegen $\$ 6$ Abs. 2 HSOG verstoßen.

Soweit das Ziel, Störungen der Baubeginns an der Okrifteler Straße zu verhindern, durch die Anordnung, ssich hinter die polizeiliche Absperrung zu begeben<, erreicht werden sollte, verstößt die Verfügung gegen den Bestimmtheitsgrundsatz des $\$ 7 \mathrm{~S}$. 1 HSOG. Es geht aus der Verfügung nämlich nicht hervor, wo diese Absperrung ist.

Selbst wenn dies den Antragstellern auf Grund von vor Ort erkennbaren Umständen zweifelsfrei möglich gewesen wäre, begegnet die Verfügung Bedenken. Polizeiliche Verfügungen dürfen nicht lediglich den Zweck verfolgen, der Verwaltung die Durchführung ihrer Aufgaben zu erleichtern ( $\$ 6$ Abs. $2 \mathrm{HSOG}$ ). Zum andern erscheint es fraglich, ob die Bestimmung eines Aufenthaltsortes in positiver wie negativer Hinsicht durch eine Polizeiverfügung überhaupt erfolgen darf. Das Grundrecht auf freie Wahl des Aufenthaltsortes nach Art. 6 der Hessischen Verfassung in Verbindung mit Art. ${ }_{42}$ GG ist nämlich durch $\S_{4}$ HSOG nicht eingeschränkt; gemäß Art. 63 Abs. I Hessische Verfassung wäre dies aber erforderlich, um einen Grundrechtseingriff dieser Art vornehmen zu können.

Die Kammer hat auch ernstliche Zweifel an der Rechtmäßigkeit des Teils der Verfügung, der die Androhung unmittelbaren $Z$ wanges enthält. Insoweit liegt ein selbständiger Verwaltungsakt vor $(\$ 12$ Hessisches Gesetz zur Ausführung der Verwaltungsgerichtsordnung). Die Durchsetzung polizeilicher Verfügungen im Wege unmittelbaren Zwanges richtet sich nach $₫ 4$ des Gesetzes über die Anwendung unmittelbaren Zwanges bei Ausübung öffentlicher Gewalt in Verbindung mit $\ 25$ Nr. 3 HSOG. Gemäß ${ }_{4}$ S. 2 des Gesetzes über die Ausübung unmittelbaren $\mathrm{Z}$ wanges ist die beabsichtigte Maßnahme, wenn die Lage es zuläßt, unmittelbar vor 
ihrer Durchführung anzudrohen. Geht man davon aus, daß die Verfügung den Antragstellern vor ihrer Vollziehung ausgehändigt oder - als Allgemeinverfügung - in sonst geeigneter Weise $\left(\$ 4^{1} \mathrm{HV}_{\mathrm{w} V f G}\right)$ dem betroffenen Personenkreis bekanntgegeben wurde, so scheint die Voraussetzung des $\$ 4$ Abs. 2 des Gesetzes über die Ausübung unmittelbaren Zwangs für die Vollziehung der Verfügung dem Wortlaut der Vorschrift noch zunächst erfüllt.

Nach der höchstrichterlichen Rechtsprechung gewährleistet jedoch Art. 19 Abs. 4 GG (vgl. den korrespondierenden Art. 2 Abs. 3 der Verfassung des Landes Hessen) nicht nur das formale Recht, den Rechtsweg zu beschreiten, sondern darüber hinaus ein substantielles Recht auf effektiven, tatsächlichen wirksamen Rechtsschutz. ,Dieser Rechtsschutzanspruch ist ein von der öffentlichen Gewalt zu beachtendes subjektives Recht des Einzelnen. Sie verletzt dieses Recht, wenn sie eine gegen den Einzelnen gerichtete Maßnahme ohne zwingenden Grund so kurzfristig anordnet und durchsetzt, daß ihm keine ausreichende Zeit zur Erlangung des gesetzlich vorgesehenen Rechtsschutzes verbleibt (BVerwG, Urteil vom 29. 10. 1963, BVerwGE ${ }_{17}, 83$ ff.). Dieser, in Art. 19 Abs. ${ }_{4}$ S. ${ }_{1}$ GG enthaltene Rechtsgedanke hat z. B. in $\$$ I $_{3}$ Abs. I S. 2 Bundesverwaltungsvollstreckungsgesetz und $\$ 69$ Abs. 2 HessVwVG seinen normativen Niederschlag gefunden.

In einer Grundsatzentscheidung des Großen Senats (Beschluß vom 11. 6. 1979, ESVGH 29, $215 \mathrm{ff}$.) hat sich der Hessische Verwaltungsgerichtshof dieser Rechtsprechung ausdrücklich angeschlossen. Unter Hinweis auf weitere, einschlägige Entscheidungen des Bundesverwaltungsgerichts sowie des Bundesverfassungsgerichts führt er aus (a. a. O. S. 220): Abgesehen von besonders begründeten Ausnahmefällen (Eilmaßnahmen bei Gefahr im Verzuge) müsse dem Pflichtigen zur Befolgung einer Zwangsmittelandrohung eine angemessene Frist gewährt werden. Diese müsse so bemessen sein, daß sie ihm die Erhebung von Rechtsmitteln und die Herbeiführung des mit der Rechtsmittelerhebung verbundenen Suspensiveffekts noch vor Beginn der Vollziehung des Verwaltungsaktes ermögliche. Insbesondere im Falle einer - wie vorliegend gegebenen - unselbständigen Androhung sei das Bedürfnis vorläufigen Rechtsschutzes evident, weil nicht cinmal die Rechtmäßigkeit der Grundverfügung feststehe. Zwar zähle bereits die Androhung von Maßnahmen der Verwaltungsvollstreckung zu denjenigen Verwaltungsakten, hinsichtlich derer Rechtsbehelfe kraft Gesetzes $(\$ 187$ VwGO i. V. m. $\$ 12$ Hess.AGVwGO) keine aufschiebende Wirkung haben.

Der Betroffene könne aber einen Antrag nach $\$ 80 \mathrm{Abs}$. 5 VwGO stellen und die Anordnung der aufschiebenden Wirkung erreichen. Dieses Recht darf ihm nicht genommen werdenc (a. a. O. S. 221). Darüberhinaus sei bei Handlungen, die der Betroffene vorzunehmen habe, regelmäßig ein Bedürfnis anzuerkennen, daß sich der Betroffene auf die Handlung vorbereite und die hierfür erforderlichen Maßnahmen treffe. Insbesondere bei Abbruchverfügungen, auch wenn sie Bestandskraft erlangt hätten, sei ein solches Bedürfnis offenkundig. Das gleiche gelte bei Zwangsräumungen. Das gelte auch dann, wenn der Betroffene gegen ein gesetzliches Verbot oder eine Strafvorschrift verstoße (a. a. O. S. 222). Bei der erforderlichen Fristsetzung handelt es sich nämlich nicht um die Duldung eines rechtswidrigen Zustandes, sondern um ein Vollziehbarkeitserfordernis.

Da bereits aus Art. 19 Abs. 4 GG folge, daß dem Pflichtigen bei Androhung die Möglichkeit gegeben sein müsse, vor Durchführung der Vollstreckung vorläufigen Rechtsschutz zu gewähren, habe die Behörde eine Rechtsschutzfrist auch dann zu bestimmen, wenn das einfache Gesetz eine solche Frist nicht vorsieht, ses sei denn, es liegen ganz außergewöhnliche Verhältnisse vor, angesichts deren selbst das verfassungsrechtliche Gebot effektiven Rechtsschutzes im Sinne von Art. 19 Abs. 4 GG übergeordneten Interessen weichen mußs (a. a. O. S. 223).

Können demzufolgen überwiegende öffentliche Belange es rechtfertigen, den Rechtsschutzanspruch des Betroffenen einstweilen zurückzustellen, um dringende Maßnahmen im öffentlichen Interesse rechtzeitig durchzuführen (BVerfGE $35,382,402$ ), so müssen diese Belange in der Begründung der Verfügung genannt sein und erkennbar zum Ausdruck gebracht werden, daß eine Abwägung zwischen den öffentlichen Belangen und dem Rechtsschutzanspruch des Betroffenen stattgefunden hat, die zu dem Ergebnis gelangte, daß diese öffentlichen Belange sich als überwiegend darstellen. Das stets gegebene öffentliche Interesse an dem Vollzug einer bestimmten Verfügung, also der Herstellung eines rechtmäßigen Zustandes, ist hier nicht ausreichend. Das gilt auch für eine Ermessensbetätigung im Rahmen der Auswahl des geeigneten Zwangsmittels. Ausführungen zu den dargestellten Fragen im Zusammenhang mit einer Abwägung zwischen überwiegenden öffentlichen Belangen und dem Rechtsschutzanspruch des Betroffenen sind in der angegriffenen Verfügung nicht enthalten.

Anhaltspunkte, die für den vorliegenden Fall ein Abweichen der Kammer von der dargelegten, eindeutigen obergerichtlichen und höchstrichterlichen Rechtsprechung (zuletzt bestätigt durch Beschluß des HessVGH vom 17.3 . 1981 - IV/TH 8/8I) rechtfertigen könnten, sind nicht ersichtlich. $[\ldots]$ « 
Diese Entscheidung konnte der Landesregierung rechtlich wie politisch nicht ins Konzept passen. Doch erklärt dies allein kaum die nachhaltige Skandalisierung durch den Innenminister, der ja nicht müde wurde, die Startbahngegner auf das rechtsstaatliche Gebot der Respektierung verbindlicher Gerichtsentscheidungen (wie insbesondere den Beschluß des Hessischen Staatsgerichtshofs vom 14./1 I . I. 1982, Az: P. St. 947, mit dem der Antrag auf Zulassung des Volksbegehrens endgültig zurückgewiesen wurde, vgl. hierzu U. Wesel, KJ H. 2/1982 S. 1 17 ff.) hinzuweisen. Zum Kernpunkt der Kritik an diesem Beschluß des Verwaltungsgerichts wurde die Tatsache, daß er gar nicht mehr hätte ergehen dürfen, weil die Polizei das Hüttendorf (mit Ausnahme der Kapelle) bereits in den Morgenstunden des 2. II. 198 I zerstört und geräumt hatte, so daß diese Entscheidung buchstäblich in der Luft hing und folglich leerlief. Nun könnte man vielleicht erwarten, daß angesichts der praktischen wie juristischen Folgenlosigkeit für die obrigkeitliche Durchsetzung des Großprojekts die erlittene gerichtliche Niederlage leichter würde hingenommen werden. Doch weit gefehlt! Offenbar sah die Administration - nunmehr in einer Art neuer Koalition von CDU-Stadtregierung und SPD/FDP-Landesregierung - darin die willkommene Gelegenheit, die unliebsamen Verwaltungsrichter in das Zwielicht mangelnder Glaubwürdigkeit zu bringen. Ausführlich nahm sich abermals die FAZ der Sache an und berichtete über die Rechtfertigungsversuche des Gerichts:

»Der Antrag der Startbahngegner ist nach Angaben des Gerichts am Montagnachmittag um I 5.45 Uhr eingegangen, zu einem Zeitpunkt also, als das Hüttendorf längst geräumt war. Weder die Antragsteller noch die Polizeibehörde als Antragsgegner, wie es im Juristendeutsch heißt, hätten die Richter über die tatsächlichen Gegebenheiten informiert, bekundet die Pressesprecherin des Gerichts, Hennelore Kohl, als Richterin der IV. Kammer selbst mit der Entscheidung befaßt. Bei den Rückfragen hätten die Verfahrensbeteiligten die Unwissenheit der Richter erkennen können, meint Frau Kohl, die den Mangel an Information darauf zurückführt, daß die Kammer vom frühen Vormittag an mit Prozeßvorbereitungen vollauf beschäftigt gewesen sei, weder Radio noch Fernsehgerät im Gericht den Zugriff zur aktuellen Lage ermöglicht hätten. Bei der Entscheidung über den Antrag des Kirchenvorstandes habe die Kammer davon ausgehen müssen, daß auf dem Gelände der geplanten Startbahn West noch nicht geräumt worden sei.«

Maliziös fährt die Zeitung fort:

„Das Verfahren und die Entscheidung der Richter aus der Adalbertstraße entbehrt nicht einer gewissen Pikanterie. [...]

Daß es sich bei den Juristen, die mit der Entscheidung befaßt waren, ausgerechnet um jene handelt, die in den Auseinandersetzungen um städtische Demonstrationsverbote von den Behörden vorgebrachte Informationen über Gewalttäter häufig als nicht ausreichend für die Begründung der Verwaltungsakte erachteten und sich lieber selbst ein Bild machten, läßt die fehlende Information vom Montag um so fragwürdiger erscheinen. Oder sollte es tatsächlich so gewesen sein, daß alle Welt, Startbahngegner wie Polizei, nur noch davon ausging, ein einziges Thema sei Gesprächsstoff und jedermann müsse wissen, was an diesem Vormittag in Flughafennähe geschehen war?

Was auch immer die Mitglieder der IV. Kammer in den Zustand absoluter richterlicher Unabhängigkeit und die Distanz von jeglicher Lebenserfahrung versetzt hat, über die RechtmäBigkeit der Räumung an sich hatte die Kammer nicht zu entscheiden.« (FAZ, 4. Ix. 198r)

Anfang Januar 1982, in Zusammenhang mit der Berichterstattung über die Beantwortung der Landtagsanfrage des SPD-Abgeordneten Kurth durch Innenminister Gries, in der dieser auf der Basis einer manipulativen Darstellung der Demonstrationsstatistik des Jahres $198 \mathrm{I}$ mindirekt zu belegen (sucht), daß Wallmann mit seiner Einschätzung in den meisten Fällen richtig gelegen habe« (FR v. 7. I. 1982, vgl. oben S. 172), kommt die FAZ unter der Überschrift »Verwunderung über Verwaltungsrichter« auf das verspätete Räumungsverbot des Hüttendorfs zurück:

"Als der hessische Innenminister Ekkehard Gries den von Verwaltungsrichtern geäußerten Vorwurf, der Frankfurter Oberbürgermeister Walter Wallmann verbiete Demonstrationen zu 
voreilig, ziemlich deutlich zurückwies (FAZ vom Mittwoch), da war das nicht der erste Strauß, den der Jurist Gries mit Verwaltungsrichtern in Frankfurt ausgefochten hat. Was er zum Beispiel davon hält, daß die IV. Kammer des Frankfurter Verwaltungsgerichts am Spätnachmittag des 2. November 198I den Räumungsbefehl für das ,Hüttendorf der Startbahngegner am Flughafen aufheben wollte, obwohl das , Widerstandsdorf ‘ bis dahin längst geräumt war, hat Gries in einer lebhaften Rede vor dem Landtag deutlich gemacht.

Nach kritischen Worten über die Justiz äußerte Gries in der Dezember-Sitzung des Landtags: ,Und dann habe ich Entscheidungen wie die des Verwaltungsgerichts in Frankfurt. Da entscheidet man nachmittags über einen Tatbestand, der morgens um 9.40 Uhr erledigt war, und zwar gewaltlos, nämlich die Räumung des Hüttendorfs. Um is Uhr telefoniert der Kammerund Gerichtsvorsitzende mit dem Polizeipräsidenten und fragt ihn über seine Zuständigkeit räumlicher Art, aber nicht über das Geschehen, über das er nachher urteilen will. Und dann wird hinterher von dem Präsidenten selbst per Telefon an die Telefonistin des Polizeipräsidiums, dessen Dienststunden längst abgelaufen waren, eine Entscheidung durchgegeben, bei der nicht einmal im Rubrum erkennbar war, welches Gericht entschieden hat. Das ist mir an jenem Abend auf den Flughafen vorgelegt worden. Wir haben gerätselt: War das Darmstadt, war das Frankfurt? Dieser Fall wird dieses Haus noch beschäftigen, weil es dazu eine Anfrage des FDP-Abgeordneten Michael Müller gibt, und ich hoffe, daß dabei die ganze Wahrheit herauskommt.

Zuvor hatte schon der FDP-Landtagsabgeordnete Wolfgang Gerhardt geäußert, was sich das Frankfurter Verwaltungsgericht da geleistet habe, habe ihn >erschüttert،. Gerhardt sagte: , Da ist telefonisch eine Entscheidung durchgegeben worden, und es war überhaupt nicht möglich, nachzufassen und zu erfahren, wer da entschieden hat, was da los war.< Warum das Gericht die Räumung verhindern wollte, als längst geräumt war, ist bekannt: die Richter waren nicht informiert. Warum dem so war, wird wohl erst bekannt werden, wenn die Antworten auf die entsprechenden Fragen des Abgeordneten Müller vorliegen." (FAZ v. 8. 1, 1982)

In der Folgezeit wird es vorübergehend ruhiger in der Kontroverse zwischen Exekutive und Verwaltungsgericht. Am I I. 2. I 982 berichtet die FR, daß der Bescheid des Verwaltungsgerichtshofes über die Dienstaufsichtsbeschwerde der Stadtverwaltung ergangen sei und es gegenüber den drei Richtern der IV. Kammer nicht zu disziplinarrechtlichen oder dienstaufsichtlichen Maßnahmen kommen werde. Stadtrat Brück erklärt, daß er derartige Maßnahmen »nicht erwartet « habe und mit dem Ausgang der Dienstaufsichtsbeschwerde "zufrieden" sei - er teile die Auffassung, "daß der >Umgang zwischen den Gewalten in Frankfurt nicht in öffentlichen Auseinandersetzungen liegen könne" (FR, I 1. 2. 198 I).

Doch diese seine (durchaus neue) Meinung sollte der Stadtrat bei nächster Gelegenheit abermals korrigieren, als der Mannheimer Morgen am 24. 8. 1982 unter den Überschriften »Verwaltungsgericht Frankfurt. Die 4. Kammer im Gerede. Eigenwillige Rechtsfindung und Informationspolitik/Der Fall Hüttendorf $\approx \mathrm{zu}$ berichten weiß:

"Als am I4. Juli wieder einmal ein teilweiser Baustopp gegen die Startbahn West des Frankfurter Flughafens gerichtlich verhängt wurde, wunderte sich bei den Eingeweihten niemand, daß diese Entscheidung von der 4. Kammer des Verwaltungsgerichts Frankfurt getroffen worden war. Der Baustopp galt der Untertunnelung der Okrifteler Straße im Zusammenhang mit dem Startbahnbau. Die 4. Kammer hatte einem Eilantrag des $»$ Bundes für Umwelt- und Naturschutz stattgegeben; wie eilbedürftig die Entscheidung war, ließ sich schon daran erkennen, daß die Umweltschutzorganisation fünf Monate zugewartet hatte, bis Bauleistungen in Millionenhöhe erbracht worden sind,, wie die Frankfurter Flughafengesellschaft den Richtern vorhielt.

Die Zeitung wiederholt sämtliche Vorwürfe, die von seiten der Verwaltungsinstanzen in der Vergangenheit gegenüber dem Verwaltungsgericht erhoben worden sind und resümiert abschließend:

"Hessische Regierungspolitiker scheuen sich weitgehend, die Rechtsprechung der 4. Kammer öffentlich zu bewerten, um keinen Anlaß für den Vorwurf zu geben, sie wollten die richterliche Unabhängigkeit unterminieren. Rechtsdezernent Brück war auf Anfrage zu einer politischen Bewertung bereit. Bei einer immer häufigeren Inanspruchnahme gegen Verwaltungsakte seien die Verwaltungsgerichte der Gefahr ausgesetzt, sagte er, die Grenzlinie zwischen Rechtmäßig- 
keitskontrolle und Eingriff in die Entscheidungsbefugnis der Verwaltung zu überschreiten. Zwischen der 4. Kammer des Verwaltungsgerichts Frankfurt und der Stadt Frankfurt stelle sich das Problem in besonderer Schärfe, weil nach Auffassung der Stadt diese Kammer dazu neigt, diese Grenzlinie bewußt zu überschreiten««.

Zwei Tage später weitet Innenminister Gries in einem Gespräch mit der Frankfurter Rundschau diese erneute Kampagne gegen die IV. Kammer des Verwaltungsgerichts aus:

"Die Kammer . . . ist nach den Worten von Gries nahe dabei, das Vertrauen der Bürger in die Unabhängigkeit der Justiz zu gefährden.

Hessens Innenminister ist gerade im Hinblick auf die Startbahnentscheidung der Vierten Kammer der Ansicht, diese Richter schienen süberhaupt nicht mehr zu wissen, wie man verkündet،. $\mathrm{Da}$ werde bei den Prozeßbeteiligten angerufen und gesagt, ssie können sich was abholen«. Gries: >Das ist juristisch keine Zustellung. W Während die Anwälte des Bundes für Umwelt- und Naturschutz (BUND) die Entscheidung schon in Händen gehabt und zu einer Pressekonferenz eingeladen hätten, hätte die Gegenpartei das Urteil noch nicht gehabt. Dazu der Innenminister. >Da hört der Spaß ein bißchen auf.»

Zahlreiche rechtliche Sünden aber hat die Kammer nach Ansicht von Gries bereits im November vergangenen Jahres begangen, als sie im Zusammenhang mit der geplanten Räumung des Hüttendorfes samt Holzkirche zu einer Entscheidung aufgerufen worden war. [...]

Dabei hätte die Kammer, so Gries, über einen Tatbestand entschieden, >der gar nicht mehr da war<. Morgens um 8.40 Uhr sei das Hüttendorf bereits gewaltlos geräumt worden, die Holzkirche sei zerlegt gewesen. Überdies sei die Kirchengemeinde gar nicht antragsberechtigt gewesen. Das habe die Kammer dann am Nachmittag dieses Tages bemerkt und sich dann sübers Telefon einwandreie Antragsteller besorgt،, Das ist eine solche Fülle von Rechtsmängeln meinte Gries, daß es zum Himmel stinkt.c Er vernachlässige dabei sogar, daß die Frankfurter Kammer gar nicht zuständig gewesen sei, sondern das Verwaltungsgericht Darmstadt.

Auch der Präsident des Frankfurter Gerichts bleibt bei diesen Vorwürfen nicht ausgespart. Präsident Neumeyer habe an jenem Novembertag gegen I 8 Uhr sdas Urteil einem Telefonmädchen beim Polizeipräsidium in Frankfurt mitgeteilt‘. Die habe es dann an den Flughafen weitergegeben. Er selbst, Gries, habe sich an diesem Abend auf dem Startbahngelände aufgehalten und dann seinen DIN-A 4 -Zettel ohne Aktenzeichen hätten die Agenturen längst die Nachricht über die Entscheidung der Vierten Kammer verbreitet gehabt. Gries: >Das war der Sinn der Geschichte.، Da könne man von ihm nicht erwarten, daß er noch Vertrauen in die Unabhängigkeit des Gerichts habe.« (FR v. 26. 8. 1982)

Mit diesen "naßforschen Äußerungen« (FR-Kommentar v. 27. 8. 1982) ist der Innenminister weit vorgeprescht. Sein vorgeblicher Verlust des Vertrauens in die Unabhängigkeit des Verwaltungsgerichts angesichts ihm politisch mißliebiger Entscheidungen und der pauschale Vorwurf einer solchen Fülle von Rechtsmängeln, „daß es zum Himmel stinkt «, übertreffen noch die Anfeindungen, denen sich die Richter der IV. Kammer von Seiten der CDU-Stadtverwaltung seit geraumer Zeit ausgesetzt sehen und offenbaren ein geradezu altpreußisch-absolutistisches Verständnis von richterlicher Unabhängigkeit. Solcher obrigkeitlicher Anmaßung von Seiten des „Verfassungsministers" tritt nun endlich der Hessische Justizminister Günther (SPD) entgegen, sicherlich ermuntert durch die von der FDP Hessen für die Zeit nach den Landtagswahlen im Herbst 1982 ohnehin aufgekündigte Regierungskoalition.

"Der hessische Justizminister Herbert Günther hat am Donnerstag entschieden die Kritik seines Kabinettskollegen Ekkehard Gries an der Verfahrensweise der Vierten Kammer des Verwaltungsgerichts Frankfurt zurückgewiesen. In Kenntnis der Umstände und nach einer Zeit der möglichen Abwägung sei die von Innenminister Gries geäußerte >barsche und unmäßige Kritik an dem Gericht schädlich für den Rechtsstaat und nicht hinnehmbarく.

$[\ldots]$

Dem hält Justizminister Günther nun entgegen, Gries hätte sehr wohl zu einer gerechten Einschätzung des Richterverhaltens kommen können, wenn er seine, Günthers Antwort, auf eine Anfrage eines Landtagsabgeordneten gelesen hätte, die sich mit den damaligen Ereignissen befaßte. 
Überdies, so Günther, sei der Gesamtkomplex bereits ausführlich in verschiedenen Sitzungen des zuständigen Landtagsausschusses ausführlich erörtert worden. Dabei sei auch darauf hingewiesen worden, daß die Kammer des Gerichts - wie in Eilverfahren nicht unüblich - mit den Verfahrensbeteiligten während der Beratung in Telefonkontakt gestanden habe.

Auch mit dem Polizeipräsidenten persönlich und mit einem weiteren leitenden Beamten des Präsidiums sei gesprochen worden. Von beiden sei nicht darauf hingewiesen worden, daß die Räumung des Hüttendorfes bereits im Gange beziehungsweise abgeschlossen sei. Vielmehr sei die Bitte des Gerichts, vor Entscheidungen über den Stoppantrag keine Maßnahmen durchzuführen, kommentarlos entgegengenommen worden.

Günther betonte, daß die Kammer an diesem Tag praktisch den ganzen Tag über in Klausur gewesen sei, um den kommenden Prozeßtag vorzubereiten, die bereits erfolgte Räumung ihr also nicht bekannt gewesen sein konnte.

Auch die vom Innenminister bemängelte telefonische Übermittlung des Entscheidungstenors an eine Telefonistin des Polizeipräsidiums sei Gegenstand ausführlicher Erörterungen gewesen. Günther: ,In Eilentscheidungen ist die telefonische Vorabinformation der Beteiligten immer dann unentbehrlich, wenn die schriftliche Entscheidung nicht mehr rechtzeitig abgesetzt werden kann.< $\mathrm{Da}$ auf verschiedene Anrufe hin eine Verbindung zum Polizeipräsidenten nicht durchgestellt worden sei, hätte ein Richter einer Mitarbeiterin im Präsidium den Tenor des Beschlusses durchgegeben. Dies mit der Bitte, den Präsidenten zu unterrichten. Das sei denn auch geschehen. $\mathrm{Da}$ der Polizeipräsident kurze Zeit darauf beim Gericht zurückgerufen habe, seien ihm dann auch die Entscheidungsgründe in kurzer Form mitgeteilt worden. >Das Gericht', so der Justizminister, ,hat daher beide Prozeßbeteiligten gleichzeitig über seine Entscheidung unterrichtet<. Die Information an die Presse sei offenbar von seiten der Antragsteller erfolgt.

An die Adresse von Ekkehard Gries gerichtet, wies Günther dringend darauf hin, sdie Verwaltungsgerichte als verfassungsmäßigen Kontrollfaktor der Verwaltung nicht in den Geruch mangelnder Unabhängigkeit zu bringen‘, (FR, 27. 8.1981)

\section{Fazit: Die Notwendigkeit einer unabhängigen Justiz}

Die verheerende Niederlage der FDP bei den Hessischen Landtagswahlen vom Oktober 1982 kann gewiß weder der Standhaftigkeit der Frankfurter Verwaltungsrichter bzw. -richterinnen noch dem Ansehensverlust der FDP als »Richterpartei« zugerechnet werden. Unbezweifelbar aber ist, daß ihre rapide Preisgabe liberaler, bürgerlich-demokratischer Positionen entscheidend zu ihrem Verlust an Glaubwürdigkeit beim Wähler und der wachsenden inneren Zerstrittenheit und Auflösung beigetragen hat. Die hier dokumentierte Konstellation, derzufolge ein FDP-Innenminister und Parteiführer als Mitglied einer SPD-Landesregierung mehr und mehr dazu übergeht, die »offensichtlich rechtswidrige« Praxis des Frankfurter CDUOberbürgermeisters im Umgang mit demokratischen Freiheitsrechten nicht nur zu billigen, sondern sich an die Spitze einer Verfassungskampagne gegen ein Gericht zu setzen, welches richterliche Unabhängigkeit noch in ihrem genuinen, die Exekutive

6 Wenig später wiederholt Innenminister Günther in einer von Gries erbetenen Stellungnahme seine Vorwürfe an die Adresse des Kabinettskollegen (vgl. FR v. 21.9. 1982) und die überregionale Presse sekundiert:

„Die weit über seine Amtskompetenz hinausgehende Richterrüge des FDP-Mannes bricht mit Grundsätzen, die zum Kernbestand des Verfassungsrechts gehören. Daß bei Polizeieinsätzen und Maßnahmen der Strafverfolgung rechtsstaatliche Prinzipien vorrangig und die Entscheidungen der Justiz zu beachten sind, darauf besteht Ekkehard Gries offenbar nur dann, wenn es ihn bei seiner Amtsführung nicht hindert. [...]

Die offenkundige Absicht des Parteichefs, mit seiner Justiz-Kritik einerseits Wähler zu gewinnen, die nicht verstehen wollen oder können, daß mehrere Gerichtsinstanzen durchaus auch mal zu verschiedenen Rechtsansichten kommen, zum anderen aber auch Druck auf die erstinstanzlichen Gerichte auszuüben, geht nach Einschätzung hessischer Justizpolitiker fehl. Von Richtern werde das, so ein Beamter des Wiesbadener Justizministeriums, sdurchweg als unerträglich empfunden, da kommt der Korpsgeist wieder durch،. Und mit solchen Angriffen, glaubt ein Verwaltungsrichter, hat sich die FDP als Richterpartei unwahrscheinlich viel versautc. (Der Spiegel, 6. 9. 1982) 
am Maßstab der Verfassung kontrollierenden Sinn wahrnimmt, ist zugleich ein exemplarisches Lehrstück für den Zerfall des parteipolitisch organisierten politischen Liberalismus in der Bundesrepublik.

Die CDU-Stadtregierung, so läßt die Statistik des Verwaltungsgerichts vermuten, hat im Verlauf der Auseinandersetzungen zurückgesteckt: Noch bis Anfang Mai 1982 wird das Gericht in sechs Eilverfahren wegen städtischer Demonstrationsverbote angerufen, wobei viermal den Anträgen der Veranstalter ganz, einmal teilweise stattgegeben wird. Seitdem - bis Anfang Dezember 1982 - ist laut Auskunft des Verwaltungsgerichts kein einziges Verfahren mehr anhängig gemacht worden und auch die Presse weiß von städtischen Demonstrationsverboten nichts zu berichten. Will man nicht unterstellen, daß auf Grund der Verbotspraxis des Oberbürgermeisters in Frankfurt Demonstrationen grundsätzlich gar nicht erst mehr angemeldet werden, so läßt dies darauf schließen, daß die Stadtverwaltung von ihrer harten Linie Abstand genommen hat.

$\mathrm{Zu}$ vermuten, daß diese Abkehr der CDU-Stadtverwaltung von ihrer wieder und wieder als »offensichtlich rechtswidrig " deklarierten Verbotspraxis auf einem Lernprozeß beruht, demzufolge sie Art. 8 GG als demokratische Fundamentalnorm, die unter keinerlei Erlaubnisvorbehalt im Einzelfall steht, prinzipiell zu respektieren bereit ist, wäre voreilig. Vielmehr wird im Gegenteil von Teilen der hessischen CDU die Strategie verfolgt, das, was ihr vor Ort angesichts des Widerstands des Verwaltungsgerichts nicht durchzusetzen gelungen ist, auf parlamentarischem Weg zu lancieren. Presseberichten zufolge ${ }^{7}$ wird auf einem Bezirksparteitag der CDU in Hessen ein Papier vorgelegt, mit dem die bisherigen Vorschläge der CDU hinsichtlich der Verschärfung des Demonstrationsstrafrechts und Versammlungsrechts um eine bemerkenswerte Variante - nämlich die hier dokumentierte Frankfurter Linie - bereichert werden. Nach den dort entwickelten Vorstellungen wird gefordert, daß eine "Vermutung der unmittelbaren Gefährdung der öffentlichen Sicherheit und Ordnung bei Aufzügen, deren Anmelder bereits in der Vergangenheit zu Demonstrationen aufgerufen haben, bei denen es im Verlauf und nach Abschluß der Veranstaltung zu nicht unerheblichen Ausschreitungen gekommen ist « (FR, 3. I I. 198 I), gelten solle. Würde es gelingen, diese Vorstellungen gesetzlich zu fixieren, so handelte es sich um einen einfachgesetzlichen Tatbestand der Grundrechtsverwirkung - und das nicht einmal auf der Basis eigener rechtswidriger Handlungen, sondern einer diffusen "Gefährdungshaftung" in Bezug auf die Friedfertigkeit aller potentiellen Demonstranten (inklusive der sagents provocateurs).

Politik ist wesentlich Interessendurchsetzung auf Kosten anderer, formuliert im $\mathrm{Na}$ men des Allgemeinwohls. Bezieht die Exekutive in solche Politik systematisch den Konflikt mit der Justiz ein, so daß diese allein die Verfassungsgarantien politischer Freiheitsrechte gewährleisten muß, so handelt es sich um Politik eines Verfassungsorgans auf Kosten eines anderen und damit der Verfassung. Die Kosten der von ihr zu verantwortenden Politik muß - jedenfalls in einer demokratischen Gesellschaft die Exekutive selbst tragen, die Risiken des politischen Prozesses aushalten. Sie auf die Judikative abzuwälzen bedeutet eine verfassungswidrige Umverteilung politischer Verantwortung. Dies deutlich gemacht, darauf trotz aller Kritik beharrt und schließlich zur Korrektur gezwungen zu haben ist das Verdienst der IV. Kammer des Frankfurter Verwaltungsgerichts.

Thomas Blanke 